\title{
Percepción de los egresados contables sobre la investigación formativa ${ }^{1}$
}

\author{
Ruth Alejandra Patiño Jacinto ${ }^{2}$ \\ Zuray Andrea Melgarejo Molina ${ }^{3}$ \\ Gloria Milena Valero Zapata ${ }^{4}$
}

Recibido: 15 de mayo de 2018

Aprobado: 4 de junio de 2018

\section{Clasificación JEL: I23, M49.}

\section{Resumen}

La investigación formativa tiene por objetivo fundamental formar y desarrollar en el estudiante habilidades distintas y un valor agregado respecto a los conocimientos disciplinares. Dado lo anterior, el presente trabajo buscó establecer la percepción que tienen los egresados de contaduría pública

1 Citar como: Patiño, R. A., Melgarejo, Z.A. y Valero G.M. (2019). Percepción de los egresados contables sobre la investigación formativa. Revista Activos, 16(30), 101-125. DOI: https://doi.org/10.15332/25005278.5062

2 Docente de la Facultad de Contaduría Pública de la Universidad Santo Tomás, Bogotá, Colombia. Líneas de desarrollo: Educación Contable, Normas Internacionales de Información Financiera, Investigación Contable. Correo electrónico: alejandrapatino@usantotomas.edu.co.

3 Profesora asociada de la Escuela de Administración de Empresas y Contaduría Pública, Facultad de Ciencias Económicas, Universidad Nacional de Colombia, Bogotá, Colombia. Líneas de desarrollo: Gestión y Desempeño en Mipymes, Contabilidad Financiera, Crecimiento Financiero Empresarial, Fracaso Empresarial. Correo electrónico: zamelgarejomo@unal.edu.co

4 Docente de la Facultad de Contaduría Pública de la Universidad Santo Tomás, Bogotá, Colombia. Líneas de desarrollo: Educación Contable, Investigación Contable, Contabilidad Ambiental. Correo electrónico: gloriavalero@usantotomas.edu.co 
acerca de los impactos de las estrategias de formación en investigación sobre sus habilidades profesionales y competencias profesionales. La metodología utilizada tuvo un enfoque cualitativo, exploratorio, de diseño narrativo. En los resultados se resaltó la percepción generalizada de una mejora en las habilidades comunicativas de los egresados, habilidades como escritura, lectura, hablar en público y capacidad de síntesis, entre otras.

Palabras clave: investigación formativa, contaduría pública, contabilidad, investigación, habilidades comunicativas.

\title{
Perception of accounting graduates about formative research
}

\begin{abstract}
Formative research has as fundamental objective to train and develop different skills and added value in students regarding disciplinary knowledge. Given the above context, this work seeks to establish the perception in Public Accounting graduates of the impact that formative research strategies have on their professional skills and competencies. The methodology has a qualitative, exploratory approach, with narrative design. Results show a widespread perception of improved communication skills in graduates, skills such as writing, reading, public speaking, ability to synthesize, among others.
\end{abstract}

Keywords: Formative research, public accounting, accounting, research, communication skills. 


\section{Introducción}

La investigación es fuente y motor del desarrollo del conocimiento en cualquier sociedad; es por ello que se ha mantenido una preocupación constante en su desarrollo, en los mecanismos de incentivos que implica, en la inversión que se le asigna y en general, por los aspectos que impacten sus resultados y, por ende, el desarrollo social y económico de cualquier entorno. En ese orden de ideas, la investigación para su análisis puede tomar diversas clasificaciones, sin embargo, para efectos de establecer categorías inmersas en las comunidades académicas, básicamente en instituciones de educación superior (IES) o universidades, se contemplan tipos de investigación objeto de análisis.

La investigación formal, la cual: "cuenta con una estructura institucional, legitimidad y apoyo financiero" (Valero y Patiño, 2012, p. 178), de igual manera, se denomina investigación propiamente dicha, es decir, que de este tipo de investigación se espera mayor impacto y resultados asociados con la rigurosidad necesaria para proponer soluciones a problemas sociales de gran envergadura. Por otro lado, la investigación formativa tiene por objeto "formar", "la investigación formativa cuyos esfuerzos se orientan a generar consciencia frente a los problemas y los retos investigativos" (Machado, Patiño y Cadavid, 2016, p. 67), a este propósito, "se fija, más que en el resultado final, en la construcción de cultura y talentos para fortalecer los espacios de comunidad investigativa mediante la formación de estudiantes e investigadores novatos" (p. 67), adicionalmente no requiere de una estructura tan elaborada y muchas veces no cuenta con recursos exclusivos, y justamente esta investigación constituyó el tema fundamental de este trabajo.

Es necesario resaltar que los dos tipos de investigación son importantes, en la medida de los objetivos que se plantean dentro de los diferentes escenarios en los que se fomentan, por lo cual Hernández (2003) plantea la necesidad de desarrollar la investigación formativa y formal de forma simultánea, en tanto son herramientas para asumir retos del entorno; frente 
a lo anterior, otro factor a considerar tiene que ver con que la investigación formativa alimenta la investigación formal, pues dentro de ella se gestan los futuros investigadores, a lo que Parra (2004) añade:

Tiene un carácter más útil y normalmente se puede resolver mejor. No se trata de que sea más fácil, por menos rigurosa o por la mayor flexibilidad metodológica que permite, sino que el valor de utilidad la hace más atractiva y motivadora, tanto para los investigadores como para sus usuarios (p. 65).

En resumen, la investigación formativa es definida como: “[...] resultado de la aplicación metódica de unos cánones lógicos desde una perspectiva o enfoque determinado, enmarcado en una conceptualización y un método que permite el análisis y la comprensión de una realidad de acuerdo con unos propósitos definidos" (Consejo Nacional de Acreditación [CNA], 1998, citado por González y Grisales, 2013, pp. 43-44). Al respecto, Restrepo (2003) señala que la importancia de la investigación formativa radica en que es un medio de la función pedagógica y aporta en la generación de conocimiento teórico; en la misma línea, Murgueitio (2011) destaca que el principal papel de la investigación formativa se centra en promover el "pensamiento propio, crítico y propositivo con la realidad indagada” (p. 92), asimismo se resalta la necesidad de obtener valoración social de este tipo de investigación.

Entonces, la investigación formativa tiene gran importancia en cualquier disciplina y para el caso de la contaduría existen adicionalmente requerimientos internacionales que básicamente buscan que se forme por competencias ${ }^{5}$ (tal es el caso de International Accounting Education Standards Board - IAESB, 2015), esto incluye la preocupación por preparar para el entorno laboral, aspecto de gran impacto, que adicionalmente ha contribuido con la inclusión de requerimientos relacionados con habilidades investigativas dentro del proceso de formación del futuro profesional contable, entre otros muchos aspectos. La incidencia de organismos

5 En general, se habla de una "adecuada combinación de conocimientos y destrezas (intelectuales, técnicos, personales, interpersonales y organizacionales) para desarrollarse como contadores profesionales" (Valero, Patiño y Duque, 2013, p. 25). 
internacionales en estas temáticas ha impulsado grandes controversias acerca de ¿para qué?, o, ¿para quién?, se forma, sin embargo, lejos de esta disyuntiva se reconoce la importancia de formar en investigación.

A partir de lo anterior, el presente trabajo tuvo como propósito establecer la percepción que tienen los egresados de contaduría pública acerca de los impactos de las estrategias de formación en investigación sobre sus habilidades profesionales y competencias profesionales; centrándose en cómo estas estrategias han aportado en la vida profesional de los egresados objeto de estudio.

En ese orden de ideas, el primer apartado del documento es la metodología, en la cual se describieron los pasos que permitieron el desarrollo de la investigación; posteriormente, se encuentra la revisión de trabajos previos de investigación e investigación formativa en contaduría, especialmente en Colombia; para luego, presentar la síntesis y análisis de los resultados de la encuesta de percepción; y por último, las consideraciones finales, que podrán ser base de discusiones futuras.

\section{Metodología}

La investigación se desarrolló a partir de una metodología cualitativa con un alcance exploratorio-descriptivo y de diseño narrativo, donde "recolecta datos sobre las historias de vida y experiencias de ciertas personas para escribirlas y analizarlas" (Hernández, Callado y Baptista, 2014, p. 504). Con ese propósito se desarrollaron las siguientes fases:

a. Búsqueda de información publicada a partir del año 2000 con el fin de establecer trabajos previos y construir el estado del arte. Las fuentes principales fueron journals internacionales tales como: Accounting education research methodology e Issues in accounting education, entre otros; para el caso de Colombia, revistas académicas, como: la Revista Contaduría y Cuadernos de Contabilidad. En estas revistas se lograron identificar artículos del tema objeto de estudio, luego de hacer una búsqueda en bases de datos en general. 
b. Se tomó como referente la categorización establecida por Patiño y Santos (2009a) acerca de estrategias de investigación formativa utilizadas en programas de Contaduría Pública: asignaturas destinadas a temas de investigación, eventos de divulgación, publicaciones, semilleros ${ }^{6}$ de investigación, y opciones de grado donde se realice trabajo escrito. Sobre estas estrategias, se planteó una encuesta (Anexo 1) a realizar por participantes voluntarios, que preferiblemente hubieran participado de algún proceso investigativo; en las preguntas realizadas sobre habilidades que desarrollaron, no se dio un listado sino que se dejó abierto para que plantearan cualquier habilidad que consideraran mejoró con sus procesos investigativos.

c. Se aplicó la encuesta a los participantes voluntarios que fueron catorce, de los cuales seis egresados pertenecían a un programa de Contaduría Pública correspondiente a una universidad privada y ocho de una universidad pública ${ }^{7}$; se graduaron del pregrado en Contaduría Pública entre el 2007 y el 2017, y sus edades oscilaban entre 22 y 33 años con un promedio de 26.6 años.

d. Con los resultados se procedió a sintetizar y analizar, generándose categorías a las habilidades que los participantes plantearon.

\section{Investigación e investigación formativa en los programas de Contaduría Pública}

Para Machado (2005), la investigación contable ha transitado por varias etapas en Colombia, las cuales son: contenidos, motivación, procesos, institucionalidad y resultados, es decir, que ha avanzado, ha cambiado de enfoque, tomando en cuenta diversos aspectos como: "la necesidad de construir comunidad investigativa como alternativa de formar los pares investigativos que propendan por el conocimiento contable científico..." (Machado, 2005, p. 27). Esto se puede llevar a cabo de distintas formas, una de ellas es a través de la investigación formativa, proporcionar la posibilidad

6 O cualquier tipo de grupo donde el objetivo sea investigar.

7 Voluntarios de la Universidad Santo Tomás y la Universidad Nacional de Colombia. 
de tomar la comunidad investigativa como estrategia pedagógica, en otras palabras, como se mencionó anteriormente, aprender haciendo.

Al respecto, Muñoz, Ruíz y Sarmiento (2015) mencionan que este tipo de investigación contribuye a que la formación sea integral y que por otra parte, se utiliza como una didáctica común; la formación integral es mencionada frecuentemente, sin embargo, la investigación es un medio para que se haga realidad, o sea, en ella los programas tienen la alternativa de proporcionar herramientas para que sus estudiantes tengan un pensamiento más amplio, distintas visiones y conocimientos interdisciplinarios, entre otros muchos aspectos.

Para Ocampo (2003), la investigación formativa presenta varias características de las que por los propósitos del presente trabajo, se resaltan algunas. El interés se basa en la formación más que en la construcción de nuevo conocimiento y alimenta la investigación que está orientada al conocimiento. En el trabajo realizado por Avendaño, Rueda y Paz (2016), se concluye que los docentes se interesan no solo por la investigación formal, sino por la formativa y que incorporan prácticas de formación en investigación de forma frecuente en su actuar como docentes, es decir, es una práctica frecuente y generalizada.

Con las ideas anteriores se puede establecer que, si bien la investigación formativa no tiene como objetivo final la obtención de resultados si se presentan, es la fuente principal de investigadores; ¿dónde aprenden?, ¿dónde se interesan por la investigación?, entonces uno de los lugares es la universidad, cuando están en el proceso de formación y la cuestión de los intereses es tan amplia, sin embargo, se podría pensar que surge de distintas estrategias o procesos intencionales o no que se desarrollan en el ámbito académico. De acuerdo con Cardona (2006), "la docencia fundamentada en la investigación permite formar a los educandos en los campos de su disciplina o profesión" (p. 39). A partir de lo anterior, se evidencia la importancia real de la investigación formativa como un proceso en el cual se impulsa el aprendizaje en muchos entornos, personal, académico y laboral, partiendo del proceso de enseñanza-aprendizaje, en últimas 
como labor de los programas de formación, en este caso en el pregrado de Contaduría Pública.

Los autores García y Botero (2014) plantean la necesidad de vincular al currículo de Contaduría Pública mecanismos que favorezcan la argumentación y la solución de problemas, entre otros; aquí los autores responden el ¿para qué?, el cual, sin embargo, puede ir más allá de lo que se proponen los programas académicos o los docentes de los mismos. El argumento se complementa de la siguiente manera:

En el caso de la contabilidad, la potenciación de un sujeto que conoce y descubre por cuenta propia, las más finas articulaciones y disonancias entre lo que plantea la teoría (marco de referencia) y la realidad, investigando hacia la configuración de nueva explicativas y comprensiones sobre lo destacado como potencial de la misma (Gracia, 2010, p. 267).

Los autores Patiño y Santos (2009a) proponen unas categorías de estrategias asociadas con investigación formativa en contaduría pública, las cuales se presentan en la Figura 1.

Acerca de las estrategias, Ríos (2013) complementa con otras, como lo son: ensayos teóricos con esquemas investigativos, club de revistas, favorecer el aprendizaje por descubrimiento y el aprendizaje basado en problemas, entre otras, las cuales pueden estudiarse en trabajos posteriores.

\section{Resultados}

\section{Asignaturas que contribuyen al desarrollo de habilidades relacionadas con investigación}

De acuerdo con Patiño y Santos (2009a), esta estrategia tiene diversos objetivos entre los cuales se encuentran: realizar una aproximación a la formulación de proyectos, desarrollar sentido crítico en los estudiantes, fortalecer el estudio de temas fundamentales como la teoría contable y 

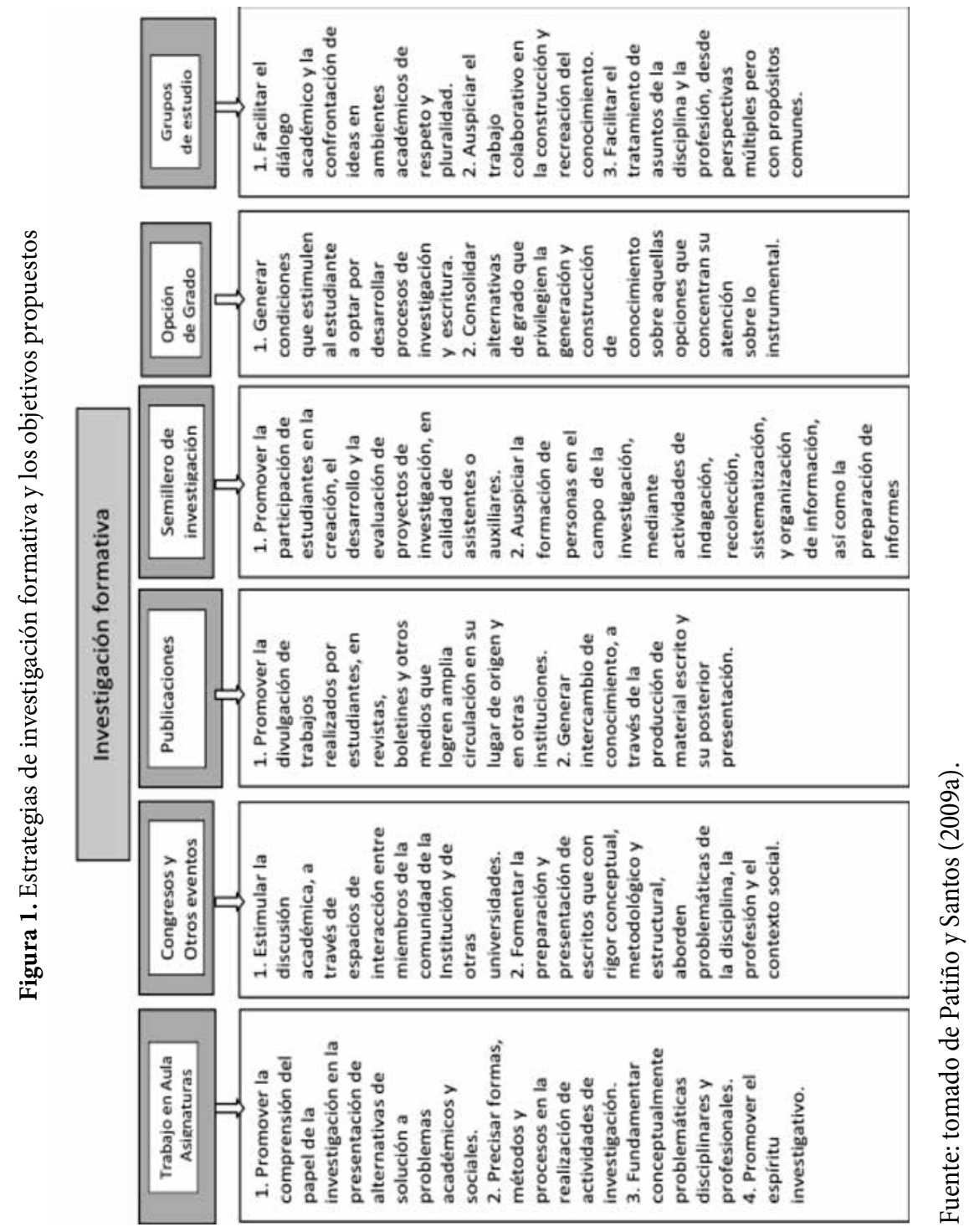
aproximarse a enfoques y técnicas de investigación como la cualitativa y la cuantitativa, entre otras.

En la pregunta de las asignaturas que identifican que les contribuyeron a desarrollar algún tipo de habilidad vinculada con la investigación, se encontró que referenciaron dos tipos de asignaturas: las que son de contenidos explícitos de investigación o habilidades comunicativas y otras que aunque son disciplinarias los encuestados perciben que les aportaron en los aspectos mencionados. Los participantes del estudio referenciaron las siguientes asignaturas de contenidos explícitos de investigación: a) Metodología de la investigación y algunas materias introductorias en la misma vía y, b) Español.

Respecto a las asignaturas que no son de contenido específico de investigación, señalaron:

- Contabilidad Ambiental

- Contabilidad Financiera

- Administración de Empresas

- Economía

- Derecho

- Antropología

- Teoría Contable

- Auditoría y Revisoría Fiscal

- Seminarios en temas de profundización como: responsabilidad social empresarial, gobierno corporativo y comités de auditoría, finanzas y presupuesto público, entre otras.

En este primer aspecto se pueden mencionar dos grandes hallazgos: a) el reconocimiento por parte de los egresados del aporte de las asignaturas destinadas a temas de investigación, ya que la pregunta estaba enfocada a determinar si percibían que contribuía y, b) lo contundente del resultado en cuanto a que la investigación formativa es definitivamente transversal, 
lo que conduce a considerar que el papel de cualquier docente es apto para aportar en el desarrollo de determinada habilidad. Los egresados mencionaron diversas asignaturas, inclusive varias que no corresponden a los componentes centrales del programa.

En un estudio previo, Patiño, Melgarejo y Valero (2017), en una muestra de 15 programas académicos de Contaduría Pública, identificaron que todos tienen asignaturas que propenden por el desarrollo de la investigación formativa, lo cual resalta la importancia de esta estrategia. Otro aspecto que se evaluó en las asignaturas fueron las habilidades que identificaron que mejoran; al respecto se incluyen las siguientes habilidades: interpretación y argumentación, redacción y elaboración de artículos, hablar en público, lectura, búsqueda de información, trabajo en grupo, referenciación y citación, y síntesis.

De los anteriores resultados se puede reflexionar que los egresados reconocieron habilidades centradas especialmente con lo comunicativo: hablar, leer y escribir, en varios aspectos como argumentar o sintetizar, pero en últimas el objetivo es el mismo. Al respecto, se evidencia que de acuerdo con (Geddes, 1998), entre otros aspectos es fundamental la búsqueda bibliográfica no solo en el desarrollo de las habilidades sino en el avance de la disciplina; lo que indica esta afirmación es el avance sobre lo que ya se desarrolló, la construcción de una comunidad académica y el avance posterior al trabajo del otro.

Adicionalmente, algo que es relevante en cualquier entorno laboral y que se constituye en saber trabajar en equipo, ya que en todos los medios es necesario y puede ser el diferencial entre el éxito y el fracaso, se añade que "la investigación dentro de los programas tiene una preponderancia que implica el desarrollo de las estrategias para promover el pensamiento y el trabajo en grupo" (Machado, Patiño y Cadavid, p. 68). El trabajo de Beverly (2016) menciona lo importante del trabajo en equipo dentro de la disciplina contable, adicionalmente recomienda que se dinamicen los cursos en los que se aporte a la consolidación de dicha habilidad, tomando como el aporte que se realiza dentro de un proceso que funciona, es decir, que 
haya coordinación entre las partes. Dentro de estas habilidades, las más mencionadas por generar efectos en los egresados se observan en la Figura 2.

En la Figura 2 se observa que la habilidad que más se recuerda en los encuestados es la de redacción y elaboración de escritos, seguida de lectura; sin embargo, cuando se les pidió que evaluaran los resultados, en otras palabras, que otorgaran una evaluación acerca de cómo impactaron en determinada habilidad las asignaturas, las de mayor evaluación fueron: capacidad de síntesis y trabajo en grupo, como se observa en la Figura 3. Entonces podría intuirse que, aunque no sean generalizadas las habilidades que presentan un impacto significativo, tales habilidades pueden desarrollarse para pocos estudiantes y generar un gran desarrollo en determinada habilidad.

Figura 2. Habilidades mencionadas por los encuestados

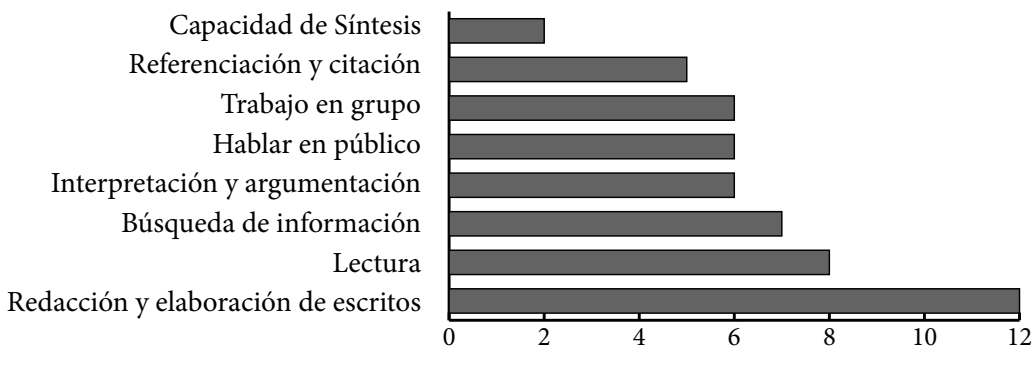

Fuente: elaboración propia a partir de las encuestas realizadas.

Figura 3. Evaluación realizada al logro de las habilidades

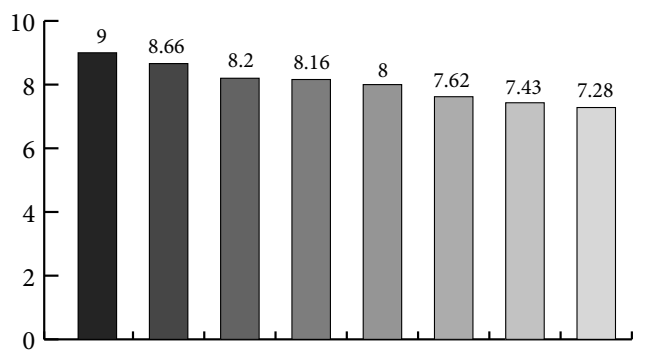

Evaluación promedio obtenida en los encuestados

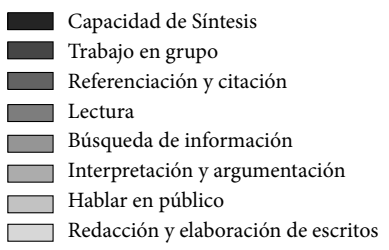

Redacción y elaboración de escritos

Fuente: elaboración propia a partir de las encuestas realizadas. 
Dehning (2015) propone que en las asignaturas se desarrolle el aprendizaje de conceptos acerca de la escritura, además de cambiar la evaluación convencional por encuestas donde se refuercen conceptos fundamentales en el aprendizaje contable; Elliott (1991) añade una preocupación no reciente de modificar el currículo, mejorando a través de la investigación, trabajando más que memoria análisis y desarrollo conceptual, complementando el ámbito laboral, frecuentemente empírico, donde se encuentran los estudiantes de contaduría pública; entonces esta propuesta se puede tomar en cuenta siempre considerando lo que ya se ha definido, la investigación formativa como estrategia transversal y complementada por otras distintas. Ordoñez (2014) plantea como error esperar que los estudiantes aprendan a investigar en un solo curso destinado a ello. Ríos (2014) en un estudio realizado, plantea que el $100 \%$ de los docentes encuestados realizan actividades dirigidas a la investigación, por lo menos algunas veces en las asignaturas, lo cual va en la misma vía de los resultados obtenidos ya que no necesariamente deben tratarse de asignaturas de investigación.

Se plantea entonces la necesidad de "incorporar en los contenidos programáticos (estructuras microcurriculares) claras formulaciones sobre los problemas fundamentales a resolver y las estrategias a emplear teniendo en cuenta los ritmos, los tiempos, el arsenal bibliográfico y las redes de comunicación" (Gracia, 2010, p. 268), lo que el profesor añade es tener en cuenta en qué ambiente se desenvuelven los actores actuales de los procesos de investigación formativa, por ejemplo, es primordial conocer cómo se realizan búsquedas en bases de datos y revistas, tanto nacionales como internacionales.

Es muy importante el papel de los docentes. Para empezar, en los cursos de Metodología de Investigación de los programas de Contaduría Pública, deben trascender los aspectos conceptuales sobre la investigación en general y sobre la investigación en contabilidad en particular, así como las cuestiones técnicas acerca de la construcción de proyectos de investigación, para ocuparse sobre los aspectos básicos de la escritura (Grajales, 2010a). El profesor Grajales resalta la importancia de aprender a leer y escribir 
en los programas de Contaduría Pública (Grajales, 2010b), otro trabajo amplía dicha preocupación a todos los actores de la comunidad académica contable (Cardozo, 2010).

En el trabajo de Patiño y Santos (2009b), se evidencia que en una muestra de programas de Contaduría Pública, el número de asignaturas que desarrollan habilidades comunicativas es de un $2 \%$ en promedio, mientras el contenido en investigación era de un $5 \%$, es decir, en total $7 \%$, un número bastante heterogéneo, donde el alto contenido es un distintivo institucional, adicionalmente todos los programas (de la muestra) tuvieron por lo menos una asignatura con contenidos explícitos de investigación.

\section{Eventos académicos}

Los eventos de investigación son importantes por ser un medio de divulgación, en palabras de Patiño y Santos (2009a) "son espacios de socialización oral de trabajos de estudiantes" (p. 28). Con base en lo anterior y frente a la encuesta, se encontró que diez de los participantes asistieron al menos a un evento académico como ponentes o expositores cuando eran estudiantes y resaltaron varios aspectos de dicha experiencia:

- Las exposiciones permiten desarrollar capacidad de interpretación y análisis.

- Se logra indagar sobre temáticas o alcances distintos a los trabajados en las asignaturas.

- Se llega a un conocimiento más profundo en temas particulares que posteriormente le han proporcionado ventajas.

- Mejora la capacidad de hablar en público.

- Aporta en conocimientos siempre y cuando se reciba la retroalimentación adecuada por parte de los docentes.

- Se presenta un aporte cultural por cuanto se realiza intercambio con otras personas, algunas veces de otros países y adicionalmente, se generan diálogos más permanentes, lo cual algunas veces termina en una relación de amistad. 
- Se aprende a tolerar las críticas, ya que en ocasiones se niega la participación en eventos, es decir, los trabajos no son aceptados.

- Proporciona bases para participar más adelante en procesos de investigación de manera un poco más formal.

- Contribuye a "manejar un público, a manejar el tiempo de exposición, a transmitir ideas de forma sencilla, a manejar una presentación como herramienta de guía y no de lectura, a dar una idea de otra persona sin olvidar mencionar que no es nuestra, a trabajar en equipo". "No puedo afirmar que en estos eventos académicos desarrollé en un $100 \%$ las habilidades mencionadas pero sí es un gran paso dentro del proceso".

- Uno de los encuestados señaló a modo de recomendación, trabajar temáticas alternativas que parecieran en un comienzo no tener relación con la profesión, como contabilidad ambiental.

De los aspectos señalados se puede resaltar que inicialmente los encuestados valoraron el proceso en los eventos académicos, ya que mencionaron diversos aspectos positivos respecto a su participación en los mismos. En cuanto a lo disciplinar, se resalta lo valioso de lograr un alcance distinto al de otros estudiantes, lo que reconocieron como ventaja posterior en el entorno laboral; en este aspecto sin embargo, uno de los encuestados mencionó la necesidad de recibir retroalimentación sobre sus trabajos para no incurrir en errores, que además se pueden socializar. Por lo demás, señalaron habilidades comunicativas y de manejo de tiempo diversas, donde es interesante indicar que el listado es bastante generoso.

\section{Publicaciones}

Dicha estrategia es la que Maldonado et al. (2007) señalan que corresponde a la visibilidad y el desarrollo científico, es decir, su importancia se basa en proporcionar resultados palpables sobre la investigación, que si bien no es el objetivo fundamental, sí genera impacto en los actores académicos participantes. Para esta estrategia diez de los catorce encuestados realizaron 
publicaciones cuando eran estudiantes, en todos los casos en revistas de divulgación o boletines. Entre las ventajas mencionadas se encuentran:

- Aporte a otros estudiantes, o sea, ellos evidencian que contribuyen al proceso de investigación de compañeros ya que incentivan la participación activa, mostrando resultados.

- Nuevamente se menciona la utilidad de ampliar el conocimiento en una temática particular.

- Mejora la forma de escribir.

- Proporciona herramientas útiles en la búsqueda de información.

- Da capacidad de síntesis.

- Presenta valor agregado en la hoja de vida.

- Suministra bases iniciales para procesos posteriores en investigación.

- Facilita el acceso a un programa de maestría.

En estos elementos se resalta que nuevamente se listan varias habilidades comunicativas, sin embargo, aparecen componentes nuevos, los cuales básicamente corresponden a mejorar su hoja de vida, lo percibieron como un valor agregado, especialmente para ingresar a programas de maestría, lo cual se constituye en un elemento de impacto más explícito, de los mencionados anteriormente. En tanto, cuando se indaga acerca de las dificultades, señalaron: en primer lugar críticas frente a los trabajos desarrollados, y en segundo lugar dificultad en la búsqueda de información.

El tema de las críticas en los trabajos desarrollados podría mencionarse en cualquier entorno donde se aspire a publicar, y posiblemente con cualquier participante (docente, estudiante, investigador experto), por lo cual, más que dificultad se podría proponer como ventaja, porque en últimas se aprende a conocer las dinámicas de las publicaciones académicas y cómo lograr los objetivos planteados.

En cuanto a la búsqueda de información, como se observa, se presenta como ventaja y como dificultad, a lo cual se le puede dar la siguiente interpretación: inicialmente es un problema no conocer cómo realizar las 
búsquedas, más con el exceso de información actual con la que se cuenta, posteriormente, cuando se realiza el aprendizaje, esto llega a ser una gran ventaja por cuanto proporciona herramientas no solo para escribir o investigar, sino para solucionar problemas.

\section{Semillero de investigación}

Corresponde a grupos de docentes y estudiantes cuyo objetivo es iniciar un aprendizaje en investigación, como su nombre lo indica, es la semilla, donde comienza todo, "son relevantes por la posibilidad de la construcción colectiva y del ejercicio mancomunado de investigadores con experiencia y otros que se encuentran en proceso formativo" (Machado, Patiño y Cadavid, p. 69).

Respecto a los encuestados, siete participaron en grupos de investigación o de semilleros, lo cual no es un dato representativo por lo que se señaló en la metodología; la encuesta se aplicó preferiblemente a egresados que hubiesen participado en procesos de investigación.

Los aspectos mencionados fueron:

- Proporciona la posibilidad de realizar intercambio y profundización de conocimientos.

- Se mejoran las habilidades analíticas y argumentativas.

- Mejoran las relaciones interpersonales y se fortalece la capacidad de trabajar en equipo.

- Se incrementa el nivel de responsabilidad.

- Se aprende a captar diferentes puntos de vista y poder llegar a un común acuerdo donde todas las partes estuvieran en línea con lo pactado.

- Mejora la relación profesor-estudiante.

- Mejoran habilidades de escritura, hablar en público, trabajo en grupo, búsqueda en catálogos bibliotecarios, base de datos, citación y referenciación de normas $\mathrm{APA}^{8}$.

$8 \quad$ American Psychological Association. 
Nuevamente se listan diversas habilidades comunicativas, dentro de las cuales se señala la citación bajo normas APA, las cuales corresponden al sistema actual exigido en la mayoría de publicaciones académicas y aunque parezca un aspecto solo de forma, contribuye a que el profesional presente sus informes de una manera adecuada y sin incurrir en plagio.

En temas personales que terminan teniendo impacto en lo personal, se relata el trabajo en equipo y la capacidad de negociación, características fundamentales en cualquier profesional y por supuesto en los contadores actuales; incluso se habla de responsabilidad, lo cual más que habilidad es una cualidad, y uno de los encuestados percibió que mejoraba al trabajar en un semillero.

\section{Opción de grado}

Corresponde a "un tipo de opción de grado que incluye algún desarrollo investigativo materializado en un escrito" (Patiño y Santos, 2009a, p. 29), es decir, es una estrategia explícita y rigurosa donde el estudiante debe presentar los resultados de un proceso de investigación en un tema específico, en otras palabras, "son una alternativa de realizar un investigación con un grado de rigurosidad, lo cual definitivamente contribuye en que el futuro profesional contable desarrolle habilidades, conocimientos y aptitudes distintas" (Machado, Patiño y Cadavid, p. 69).

Cinco encuestados desarrollaron opción de grado por trabajo escrito, solo uno de ellos señaló la motivación por interés en un tema específico, estos participantes resaltaron los siguientes logros:

- Capacidad de interpretación.

- Profundización en temáticas profesionales (disciplinares).

- Mejora la redacción y la estructuración de textos.

- Genera habilidades para recolección de datos, búsqueda bibliográfica y análisis de información.

- Se exige proponer soluciones a problemas. 
En esta última estrategia, la única habilidad adicional que percibieron los egresados fue la capacidad de proponer solución a los problemas, lo cual es un gran aporte para el profesional contable, ya que en el día a día, en las diferentes áreas de ejercicio profesional, surgen dificultades donde el contador puede aportar dando soluciones, lo cual hace la diferencia en las organizaciones.

\section{Consideraciones finales}

Es evidente la importancia de la investigación formativa, sin embargo, el presente trabajo indagó sobre la percepción de los egresados de Contaduría Pública respecto al impacto de distintas estrategias utilizadas en los programas de formación. Con ese propósito se concluye que el proceso de formación investigativa se percibe y se debe realizar de forma transversal, por cuanto los egresados consideran que además de asignaturas como Metodología de Investigación, también aportan asignaturas disciplinares e interdisciplinares, a partir de lo cual surge una preocupación nueva, centrada en si de los docentes la totalidad de ellos están conscientes del papel activo que desempeñan en el desarrollo de competencias y habilidades investigativas en sus estudiantes, ya que por lo menos se debe cuestionar el tipo de lecturas que se abordan dentro de las asignaturas, qué tipo de lectura se desarrolla, entre otros aspectos, pero no solo los profesores de investigación sino todos. En este sentido, Kent, Wilson, Ravenscroft y Rebele (2009) reconocen que muchos docentes no son investigadores activos aunque tienen interés en la educación e investigación contable, siendo esto una oportunidad para aprender.

Otro aspecto a señalar es una falencia, es decir, un aspecto que se resalta por su ausencia y es el tema de manejo de una segunda lengua, ya que si bien es importante dominar bibliografía en español, en el entorno actual se produce bastante material, especialmente en inglés, el cual, si no es utilizado, implicaría una visión parcial de un tema determinado en tanto no se contemplan los desarrollos académicos en otros contextos. 
Al mismo tiempo se evidencia el desarrollo en habilidades distintas a las netamente profesionalizantes, especialmente las comunicativas, aspecto que fue reconocido por los egresados como una importante ventaja que aparece en la totalidad de las estrategias mencionadas. Adicionalmente a las habilidades comunicativas, se enuncian habilidades personales tales como relaciones interpersonales, capacidad de negociación, e inclusive en un caso mejoramiento en la relación docente-estudiante; esto es, se percibe como una ventaja frente a los que no han participado en procesos de investigación formativa.

Por otro lado, los egresados mencionaron que ganan en cuanto a elementos culturales como: conocer diferentes lugares, conocer personas de otros países, lograr un diálogo permanente, o desempeñarse en escenarios distintos al rutinario, aspectos que definitivamente cambian el perfil del contador, el cual cada vez se enmarca menos en un solo entorno, presionándolo a ser versátil y conocedor de su contexto. Una contribución adicional de este tipo de procesos está dada por el conocimiento de nuevos temas, los cuales en un principio los egresados ni siquiera los reconocían como relacionados con la disciplina, tal es el caso de la contabilidad ambiental, esto amplía la visión del futuro profesional frente a la riqueza temática y alternativas de desempeño que tiene la contaduría.

Para terminar, "el estudiante debe partir del conocimiento de la realidad, su concepción, su crítica y la formulación de problemas inherentes, para llevarlo por medio de la investigación a planos científicos que en la proposición de soluciones forjen nuestra identidad política" (Grupo de estudios contables "Nosotros", Universidad Nacional de Colombia, 1988, p. 68), esto contribuye a generar soluciones sociales asociadas con la disciplina.

\section{Referencias}

Avendaño, W., Rueda, G. y Paz, L. (2016). La investigación formativa en las prácticas docentes de los profesores de un programa de Contaduría Pública. Cuadernos de Contabilidad, 17(43), 157-182. 
Beverly, R. (2016). Teamwork from accounting graduates: what do employers really expect? Accounting research Journal, 29(3), 348-366.

Cardona, J. (2006). Reflexiones en torno a la relación docencia-investigación en materia contable. En J. Cardona, y M. Zapata, Educación contable: antecedente, actualidad y prospectiva (pp. 43-55). Medellín, Colombia: Universidad de Antioquia.

Cardozo, B. (2010). Enfoque de la competencia comunicativa como una alternativa de solución al problema de incomunicación en el proceso contable. En C. d. Pública, I Encuentro Nacional de Profesores de Contaduría Pública (pp. 479492). Bogotá D. C., Colombia: Corcase Editores.

Dehning, S. (2015). Learnings logs: Incorporating writing-to-learn assignments into accounting courses. Issues in accounting education, 30(2), 79-104. DOI: https://doi.org/10.2308/iace-50980

Elliott, R. (1991). Accounting education and research at the crossroad. Issues in Accounting Education, 6(1), 1-8.

García, M. y Botero, G. (2014). Entre la vida académica y la práctica profesional: problemas en la formación de los contables colombianos. Contexto, (3), 174-179.

Geddes, B. (1998). The development of accountancy education, training and reserach in England: method and methodology. Accounting Education (7), S171-S178.

González, E. y Grisales, L. (9 de octubre de 2013). Acerca de la investigación formativa como concepto transversal para los currículos de pregrado de la Universidad de Antioquia, informe final de investigación. Recuperado de http://www.uptc. edu.co/eventos/2013/cf/cipni/memoria/memorias_preliminar_cip3.pdf

Gracia, E. (2010). La investigación contable en el acto educativo. Contaduría, (57), 255-269.

Grajales, J. (2010a). La escritura de anteproyectos de investigación en un ambiente de no-nolectores. El caso del Curso de Metodología de la Investigación Contable en la Universidad del Valle Sede Norte del Cauca. Contaduría, (57), 145-159.

Grajales, J. (2010b). La importancia de enseñar a los estudiantes de contaduría pública a leer y a escribir. En C. d. Pública, I Encuentro Nacional de Profesores de Contaduría Pública (pp. 331-341). Bogotá D. C., Colombia: Corcase Editores. Grupo de estudios contables "Nosotros", Universidad Nacional de Colombia. (1988). La actitud del estudiante como factor de la investigación contable. Contaduría, 12, 51-70. 
Hernández, C. (2003). Investigación e investigación formativa. Nómadas, 183-193. Hernández, R., Callado, C. y Baptista, P. (2010). Metodología de la investigación. México D. F., México: McGraw Hill.

International Accounting Education Standards Board - IAESB. (2015). Handbook of International Education Pronouncements. Recuperado de https://www.ifac. org/publications-resources/2015-handbook-international-education-pronouncements

Kent, P., Wilson, R., Ravenscroft, S. y Rebele, J. (2009). The role of accounting education research in our discipline an editorial. Issues in Accounting Education, 24(2), 123-130. DOI: https://doi.org/10.2308/iace.2009.24.2.123

Machado, M. (2005). Investigación contable formativa para construir comunidad investigativa. Revista Asfacop, 8, 21-38.

Machado, M., Patiño, R. A. y Cadavid, Y. (2016). La investigación contable en Colombia. En M. Machado, Caminos Contables (pp. 49-85). Medellín, Colombia: Universidad de Antioquia.

Maldonado, L., Landazábal, D., Hernández, J., Ruíz, Y., Claro, A., Vanegas, H. y Cruz, S. (2007). Visibilidad y formación en investigación. Estrategias para el desarrollo de competencias investigactivas. Revista Studiositas, 2(2), 43-53.

Muñoz, S., Ruíz, G. y Sarmiento, H. (2015). Didácticas para la formación en investigación contable: una discusión crítica de las prácticas de enseñanza. Revista Investigación y Reflexión, 23(1), 53-80.

Murgueitio, M. (2011). La investigación formativa como eje dinamizador hacia el pensamiento crítico del ejercicio contable. Revista Gestión y Desarrollo, $8(1), 79-94$.

Ocampo, E. (2003). La investigación formativa frente a los requerimientos del decreto 939 de mayo de 2002 para los programas de Contaduría Pública. Revista Asfacop, 7, 47-62.

Ordoñez, S. (2014). Inquietudes sobre la formación de contadores públicos: ¿por qué eligen la contaduría pública como opción profesional, qué entienden por contabilidad y sobre qué les gustaría investigar? Análisis descriptivo de 76 respuestas e invitación a una formación te. Contaduría(65), 143-172.

Parra, C. (2004). Apuntes sobre la investigación formativa. Educación y educadores, 7, 57-55. 
Patiño, R. A. y Santos, G. (2009a). La investigación formativa en los programas de Contaduría Pública, caso Colombia. Capic Review, 7, 23-34.

Patiño, R. A., Melgarejo, Z. A. y Valero, G. M. (2017). La investigación formativa en los planes de estudio. International Business \& Economics Research Journal IBER.

Patiño, R. y Santos, G. (2009b). Planes de estudio de contaduría pública en Colombia y las propuestas de formación profesional. Revista Internacional Legis de Contabilidad \& Auditoría, 131-163.

Restrepo, B. (2003). Investigación formativa e investigación productiva de conocimiento en la universidad. Nómadas, 195-202.

Ríos, R. (2013). La formación para la investigación en los programas de Contaduría Pública. Revista Colombiana de Contabilidad, 1(2), 11-42.

Ríos, R. (2014). La formación para la investigación y su incidencia en la cualificación académica de los estudiantes de contaduría en Colombia. Teuken Bidikay, 5, 99-122.

Valero, G. y Patiño, R. (2012). Los grupos de investigación contable reconocidos por Colciencias. Cuadernos de Contabilidad, 13(32), 175-201.

Valero, G., Patiño, R. y Duque, O. (2013). Competencias para el programa de Contaduría Pública: una aproximación conceptual. Contaduría, 62, 11-36. 


\section{Anexo A}

Preguntas del instrumento utilizado para la recolección de información

1. ¿Cuáles asignaturas de su pregrado recuerda con contenido de investigación o desarrollo de habilidades comunicativas o de búsqueda de información, como lectura, escritura, síntesis, interpretación, hablar en público, trabajo en grupo, búsqueda en catálogos bibliotecarios, base de datos, citación y referenciación en determinadas normas, entre otras?

2. Explique qué habilidad pretendía desarrollar cada una de las asignaturas mencionadas en la pregunta 1.

3. Si tuviera que dar una calificación al logro de esos objetivos propuestos, ¿cuál sería? (Entre 0 y 10 ) siendo 10 el total del logro.

- Habilidad 1 (mencione cuál es la habilidad y su evaluación numérica entre 0 y 10$)$

- Habilidad 2 (mencione cuál es la habilidad y su evaluación numérica entre 0 y 10$)$

- Habilidad 3 (mencione cuál es la habilidad y su evaluación numérica entre 0 y 10$)$

- Habilidad 4 (mencione cuál es la habilidad y su evaluación numérica entre 0 y 10$)$

- Habilidad 5 (mencione cuál es la habilidad y su evaluación numérica entre 0 y 10$)$

4. ¿Participó cuando era estudiante en eventos académicos como congresos, simposios, encuentros, entre otros, como ponente o expositor?

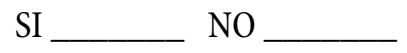


5. Si la pregunta anterior es afirmativa, por favor comente ¿cómo fue la experiencia y cuál es el aporte que le proporcionó a nivel personal? Además, describa su opinión sobre la importancia y pertinencia de este tipo de eventos en el desarrollo sus habilidades comunicativas.

6. ¿Realizó publicaciones en una revista cuando era estudiante? Si es así, cuéntenos por favor la experiencia en cuanto al aporte que le proporcionó, así como algunas de las dificultades a las que se enfrentó en el proceso.

7. ¿Perteneció a un grupo de semillero de investigación cuando era estudiante? Si es así, cuéntenos por favor la experiencia en cuanto al aporte que le proporcionó, ¿mejoró alguna habilidad de las mencionadas con anterioridad u otra diferente?

8. ¿Realizó algún trabajo escrito como opción de grado? Si es así, cuéntenos por favor la experiencia en cuanto al aporte que le proporcionó, ¿ ¿mejoró alguna habilidad? Adicionalmente indique las principales razones que motivaron dicha selección. 